\title{
Review of: "Tissues, not blood, are where immune cells function"
}

\author{
Cleo Goyvaerts $^{1}$ \\ 1 Vrije Universiteit Brussel
}

Potential competing interests: The author(s) declared that no potential competing interests exist.

Prof. Donna L. Farber from the Department of Microbiology and Immunology at Columbia University Irving Medical Center, makes a crystal-clear plea for more immunological research into human tissues instead of basic blood samples.

Humans have ample blood, which can be collected regularly. Therefore, blood samples are mostly used to assess the patient's immunological profile. However, several subsets of 'white blood cells' are very scarcely present within blood while they display a plethora of subsets and functions in different tissues.

Macrophages are a textbook example of this issue. Moreover, obtained results from human blood samples are often complex to relate to findings from preclinical mouse studies. These often focus on the immunological assets within the tissues of interest, while murine blood samples are less explored owing to the tiny volumes obtained.

In this commentary, Prof. Farber elegantly points out three recommendations that could ameliorate more relevant immunological research. First, clinicians and immunologists should find more opportunities to pair the collection of human blood and tissue samples. Second, biobanks should store more frozen tissues and secretions of immune cells and soluble factors. Third: any type of collaboration between immunologists and/or clinicians should be supported and streamlined. Among others by providing more training clinicianscientists in immunology laboratories and encouraging more joint research projects that extent beyond acquiring samples.

As an immunologist in the field of lung cancers with complex solid tumor microenvironments, I fully concur with Prof. Farber's plea. More collaborations between researchers and clinicians will lead to crosspollination with a better understanding of immunity next to more relevant tissue-oriented and translatable research into the convoluted world of immunology. 